\title{
Combinatorial-Entropy-Driven Aggregation in DNA-Grafted Nanoparticles
}

\author{
Francesco Sciortino, * Yugang Zhang, Oleg Gang, and Sanat K. Kumar
}

Cite This: ACS Nano 2020, 14, 5628-5635

Read Online

\section{ACCESS 1}
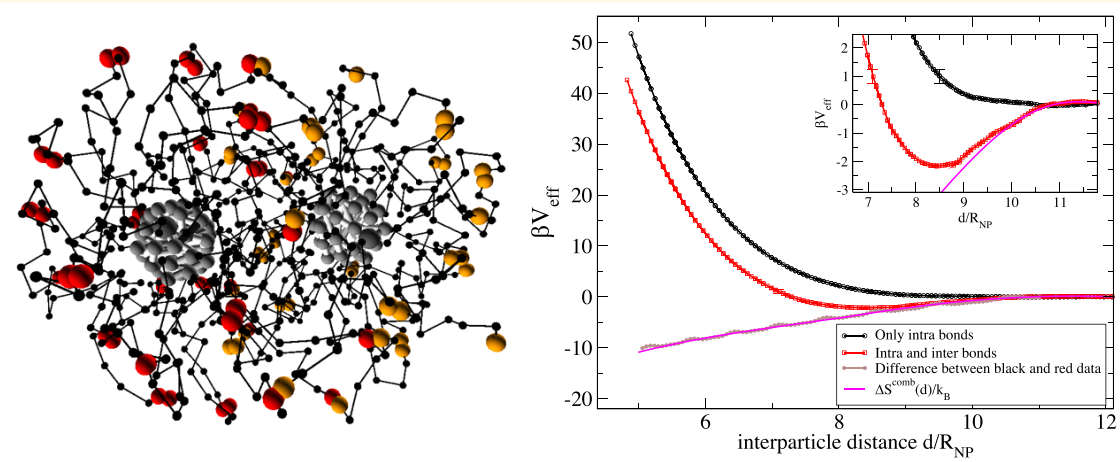

ABSTRACT: We use computer simulations and experiments to study the interactions between nanoparticles (NPs) grafted with self-complementary DNA strands. Each strand ends with a sticky palindromic single-stranded sequence, allowing it to associate equally favorably with strands grafted on the same particle or on different NPs. Surprisingly we find an attractive interaction between a pair of NPs, and we demonstrate that at low temperature it arises purely from a combinatorial-entropy contribution. We evaluate theoretically and verify numerically this entropic contribution originating from the number of distinct bonding patterns associated with intra- and interparticle binding. This entropic attraction becomes more favorable with decreasing inter-NP distance because more sticky ends can participate in making this choice.

KEYWORDS: DNA, grafted colloids, entropic effects, crystallization, simulation

\begin{abstract}
T $\mathrm{t}$ is well-understood that colloids or nanoparticles (NPs) grafted with single-stranded DNA can assemble into crystal structures when they are mixed with particles with the complementary DNA sequence at low temperatures. ${ }^{1-6}$ This well-explored fact is the basis of a rapidly evolving field of research pairing experimental studies with theoretical and numerical investigations. ${ }^{7-13}$ The predominant theoretical idea in this field is to use the free energy of DNA hybridization to predict the NP-NP interaction potential. This approach can, for example, yield the ground-state (free) energy of different lattices. We can then assign the one with the lowest free energy as being the crystal structure spontaneously assumed by the NPs. An excellent implementation of this idea is the continuum contact model, which uses purely nearest-neighbor energetic interactions to decide between $\mathrm{CsCl}, \mathrm{AlB}_{2}$, and $\mathrm{Cr}_{3} \mathrm{Si}$ structures. ${ }^{14}$ While Biancanello et al. ${ }^{15,16}$ have delineated the underlying energetics associated with such pairings from computer simulations, they recognize that these are effective interactions that naturally include entropic terms. More sophisticated approaches, such as a liquid-state model of Vo
\end{abstract}

et al., ${ }^{17}$ go beyond the prediction of simple lattices to predict the formation of more complex forms found experimentally. Recently, Travesset and co-workers ${ }^{18}$ and Lequieu et al. ${ }^{19}$ have used computer simulations to understand this assembly process in detail and have begun to stress the importance of entropic factors in this assembly.

Entropy plays a very important role in colloidal self-assembly (for recent reviews see refs 20,21). Our focus in this article is to emphasize the less-known role played by combinatorial entropy in defining the assembly of nanoparticles grafted with palindromic (self-complementary) DNA sticky sequences, i.e., sequences that are able to pair with identical sequences, grafted

Received: December 24, 2019

Accepted: May 6, 2020

Published: May 6, 2020 

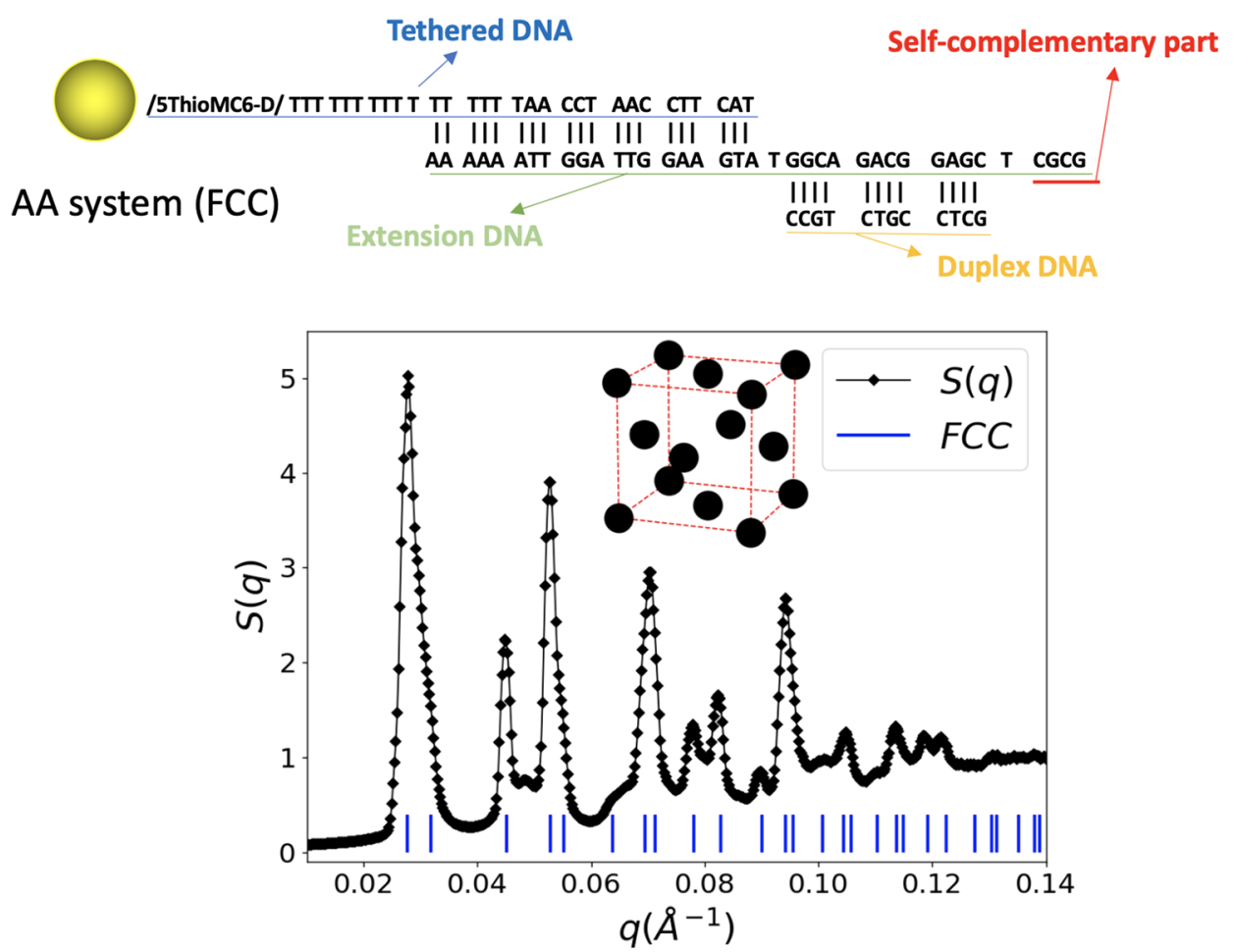

Figure 1. (Top) Schematic illustration of the DNA-grafted NPs studied in this work. (Bottom) The measured structure factor, $S(q)$, reveals the assembly of a superlattice formed by gold nanoparticles grafted with self-complementary DNA. The structure is indexed as a facecentered-cubic ( $\mathrm{fcc}$ ) lattice, where calculated diffraction lines are shown in blue and the unit cell is shown in the inset. The index of the first eight diffraction lines corresponds to (111), (200), (220), (311), (222), (400), (331), and (420) planes. The lattice constant of the observed fcc structure is $\sim 39.4 \mathrm{~nm}$.

either on the same particle or on a different one. In the case of an isolated particle all of the DNA sequences are expected to be paired among themselves at low enough temperatures, giving rise to ideally inert particles. One would then expect that NPs grafted with palindromic DNA sticky sequences should not bind, since there is no energetic gain that can compensate the loss of translational entropy upon interparticle binding. Hence the naive expectation would be that there should be no attractive interactions between two NPs as they are brought from infinite separation toward contact.

In contrast to these ideas, experiments show that these NPs organize into crystalline arrays, implying that there is a favorable interaction between the NPs, one that is strong enough to drive the particles to self-assemble into a facecentered-cubic (FCC) crystal (see Methods section for details). Such superlattices were created from $\sim 10 \mathrm{~nm}$ gold nanoparticles using DNA-mediated assembly in a four-step procedure. First, the nanoparticles were tethered with a 30base single-strand DNA in a $10 \mathrm{mM}$ phosphate buffer with 250 $\mathrm{mM} \mathrm{NaCl}$. The details for the functionalization of DNA were described in ref 22. Second, a 38-base extension DNA was introduced to the solution to form a 20-base duplex sequences with the tethered DNA. The extension DNA includes three blocks (see Figure 1 top). At one end, there is a 20-base block, which hybridizes with the corresponding complementary part of the tethered DNA. In the middle a 12-base part is hybridized with an additional added complementary singlestrand DNA to form a duplex. At the other end, there is a 4base (CGCG) block, which acts as the sticky end. There is one base between each of the blocks. In the third step, a few hours after the second step, the nanoparticles were assembled into amorphous aggregates at room temperature. Finally, the assembled structure was crystallized by annealing at $38{ }^{\circ} \mathrm{C}$ (the melting temperature is $\sim 40{ }^{\circ} \mathrm{C}$ ) for a few hours. The structure factor, $S(q)$, obtained from a small angle x-ray scattering measurement, for the resulting structure in Figure 1 clearly show that colloids grafted with palindromic DNA sequences assemble into crystalline lattices (in this case an FCC lattice), evidently due to inter-NP binding, as discussed above.

To understand these results, here we use computer simulations of a simple coarse-grained model to demonstrate in a quantitative way that a combinatorial entropic attraction arises when DNA sticky sequences are offered the possibility to decide to form bonds between chains from the same NP or from other NPs. We quantify this attraction and show that it can reach several $k_{\mathrm{B}} T$ units, large enough to compensate the unfavorable entropy associated with the overlap of the grafted layers ("brushes") on adjacent NPs. This competition results in a minimum in the effective potential between two adjacent NPs, which can be strong enough to cause the NPs to aggregate (i.e., form either a disordered liquid-like state or even an ordered solid under appropriate conditions). This combinatorial contribution, which appears when the binding sequence is palindromic (or when two complementary binding sequences are grafted on the same $\mathrm{NP}^{23,24}$ ), is different from previously discussed entropic terms for DNA-coated colloids arising from restricted freedom of motion of the sticky ends upon binding or from the different ways in which bonds can form between two nearby colloidal surfaces. ${ }^{25,26}$ We performed molecular dynamics simulations on two NPs, each of radius $R_{\mathrm{NP}}=1$ (which defines the elementary length scale in the problem). The NPs were modeled as isospheres, ${ }^{8}$ created by splitting each triangle of a regular polyhedron into four smaller 
triangles, resulting in a tessellated sphere of 42 sites. A chain of seven monomers was attached to each of these 42 sites. An additional end sticky bead (at the free end of the chains), being "sticky" to all other sticky beads, mimics the palindromic DNA binding sequence. The chains were described by the KremerGrest model, where adjacent monomers were connected by FENE springs. Interactions between the non-sticky beads (and between all chain monomers and the NPs) were modeled by a truncated, shifted LJ potential that was repulsive everywhere. The interaction between sticky monomers was modeled by a short-range potential with a minimum at $r=0.3$ with a deep well such that all sticky ends pair at the studied temperature. The parameters of all interaction potentials are reported in the caption of Figure 2 and discussed in more depth in the
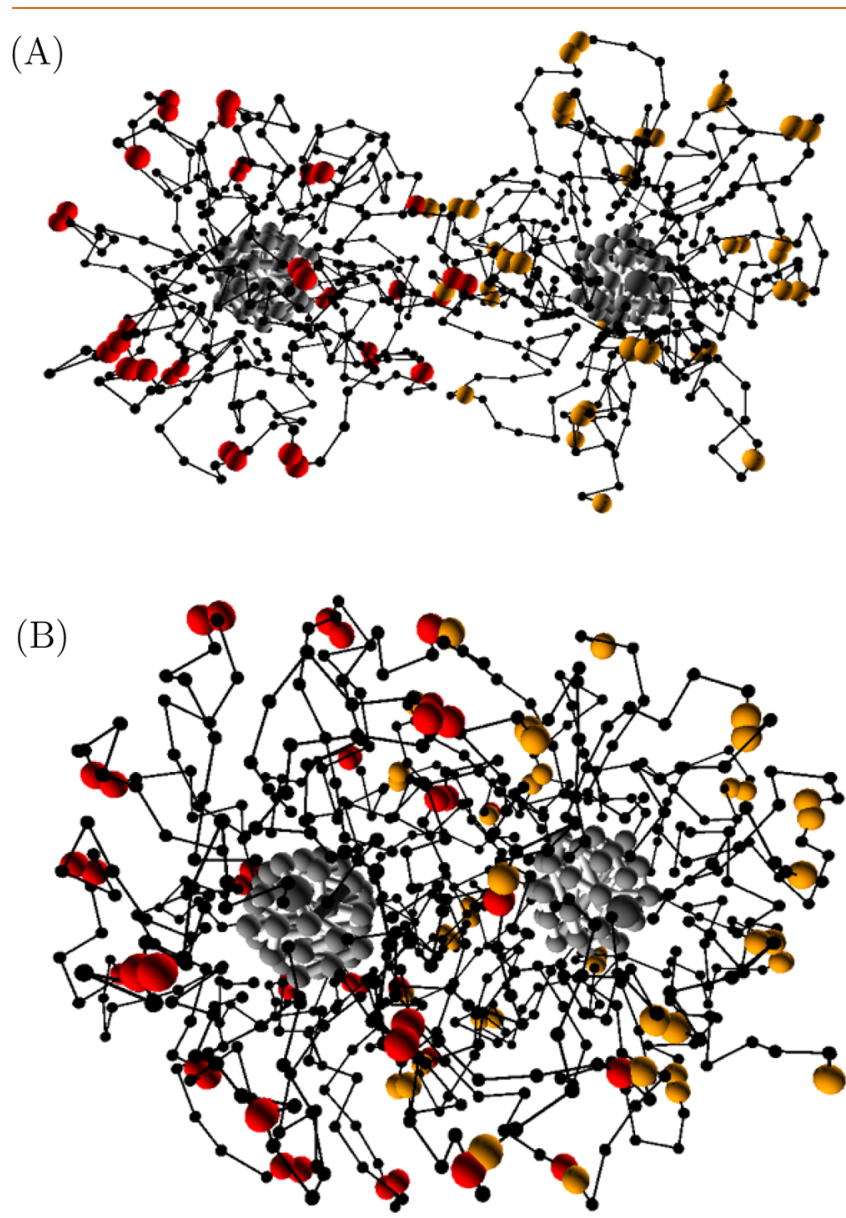

Figure 2. (A and B) Snapshots of two NPs with grafted chains, where the sticky ends on the two NPs are colored red and orange, respectively. The core is colored in gray, while the nonsticky chain monomers are in black and made artificially smaller to emphasize the sticky ends. The two NP centers are separated by (A) $d=8 R_{\mathrm{NP}}$ and (B) $d=4 R_{\mathrm{NP}}$. Notice the increased number of red-orange pairs in $(B)$.

Methods section. A repulsive three-body term was then added to enforce the single-bond per sticky site condition and, more importantly, to facilitate the swapping of partners for the sticky ends while leaving the energy of the system essentially constant. ${ }^{27,28}$ Different from other bond-swapping schemes $^{29,30}$ the three-body algorithm can be naturally encoded in molecular dynamics. This three-body term is central to realizing equilibrium in terms of bonding when the bonding energy is much larger than $k_{\mathrm{B}} T$, because otherwise a pair of bonded DNA strands would essentially never unpair and find other partners in a normal MD simulation. Since we begin with two NPs separated by large distances and then bring them closer, such partner exchange moves are critical to establishing the equilibrium relative population of intra-NP $v s$ inter-NP DNA bonds.

Before discussing the results we note that we neglect any bending contributions to the system free energy in the present model. These factors could be particularly important to the different geometries that have to be adopted by the chains to form intra- $v s$ inter-NP bonds. While this approximation will likely overpredict the fraction of intra-NP bonds, we justify it by the presence of unpaired bases ("spacers") in the DNA sequence (see Figure 1). The very short persistence length of single-stranded DNA significantly reduces the bending freeenergy difference between intra- and interparticle bonds (besides mechanically decoupling the double helix generated by the binding process from the rest of the DNA sequence).

\section{RESULTS AND DISCUSSION}

To calculate the effective interaction potential (the potential of mean force, PMF) between two NPs, we use umbrella sampling, ${ }^{31}$ spanning an interval of distances from the closest distance at which the two particles feel each other down to distances for which $V_{\text {eff }}(d)=50 k_{\mathrm{B}} T$. We use a harmonic potential with the mean separation between the NPs and its width being the two control variables. We used 38 different mean separations to systematically calculate the PMF as a function of the interparticle distance $d$. The resulting PMF is shown in red in Figure 3 as a function of the separation between the centers of the two particles. For distances greater than $\sim 11$, the potential is effectively zero, implying that there are no interactions between the two NPs. For shorter distances

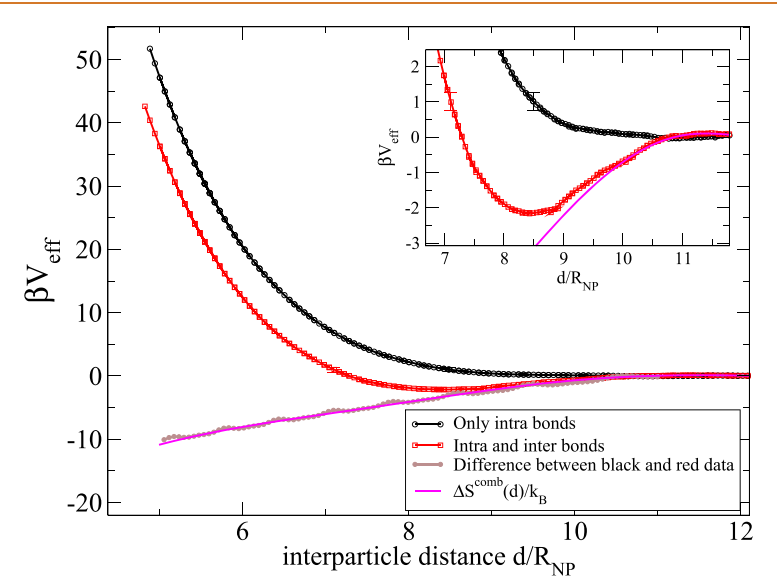

Figure 3. Calculated potential of mean force as a function of the center-to-center distance $d$ between two NPs (red line) grafted with polymeric chains ending in a (palindromic) sticky end. The black line - which only captures the contribution from brush entropy - results by preventing any pairing of sticky ends across the two NPs, while allowing the chain ends on a single NP to pair. The brown points joined by the brown line shows the difference of the total PMF and the repulsive brush contribution (black line minus red line). The magenta curve is calculated according to eq 1 . Note that the good agreement of these last two estimates illustrates that we have a full understanding of the two-body PMF. The inset shows an enlargement of the same curves, to highlight the strength of the attraction arising from combinatorial entropy. Typical error bars are also shown. 
the potential is negative (favorable) with a minimum value of ca. $-2 k_{\mathrm{B}} T$ for a separation of $\sim 8$. Below this distance the particles repel each other, with the potential increasing to $\sim 50 k_{\mathrm{B}} T$ for a separation of $\sim 5$. To highlight the effect of the interparticle bonding, we repeat the above calculation but explicitly prevent the bonding of sticky ends from distinct NPs, while confirming that the total number of bonds between sticky ends is identical in both cases. This is technically performed by turning off the sticky-sticky potential between sticky sites belonging to distinct particles. This calculation provides a measure of the brush distortion entropy contribution, which is found to be always repulsive, stressing that this contribution is always unfavorable (black line in Figure 3). The difference between the two calculated effective potentials provides a measure of the added entropy afforded by the sticky ends having a choice of picking ends from the same NP or from the second NP to pair.

To provide evidence that this binding entropy difference arises from the ability to bond with sticky ends from the same vs distinct NPs, we theoretically evaluate the combinatorial contribution originating from the pairing of the sticky ends. We define $N(r)$ as the number of sticky ends of each particle that, for each NP separation $r$, could bind with sticky sites of the distinct partner particle (i.e., those sticky ends that can reach a potential partner on the other NP when the NP centers are separated by $r$ ). If only intraparticle bonds are allowed, then the number of possible different bonding patterns is $[(N(d)-1) ! !]$ for each NP (or $[(N(d)-1) ! !]^{2}$ for the two NPs). Here the double factorial !! originates from the fact that each bond eliminates two sticky sites from the ensemble of the $N(d)$ sticky sites. If interparticle bonding is instead allowed, the expression is identical, but $N(d)$ must now be substituted with $2 N(d)$. The entropy difference arising from the increase in the number of possible bonding patterns when interparticle bonding is allowed can thus be expressed as

$$
\frac{\Delta S^{\mathrm{comb}}(d)}{k_{\mathrm{B}}}=\ln \left[\frac{(2 N(d)-1) ! !}{[(N(d)-1) ! !]^{2}}\right]
$$

This expression embodies our estimate of the extra combinatorial entropy because of the ability to bond with sticky ends from the same $v s$ the second NP. While a theoretical estimate of $N(d)$ is not straightforward, the number of bonds $N_{\mathrm{b}}^{\text {inter }}$ that can form between sticky ends on different NPs for each separation $r$ is a quantity available from the numerical simulations; $N_{\mathrm{b}}^{\text {inter }}$ provides an exact measure of $N(d)$. Indeed, assuming random mixing, $N_{\mathrm{b}}^{\text {inter }}=N(d) / 2$ (out of the $N(d)$ bonds, one-quarter are between sticky ends of the first particle, one-quarter are between sticky ends of the second particle, and half of them are interparticle bonds). Figure 4 shows $N_{\mathrm{b}}^{\text {inter }}$ as a function of $d$. It thus becomes possible to precisely evaluate the importance of the radial dependence of the combinatorial entropy of eq 1 . The stabilizing combinatorial entropy contribution to the PMF is plotted in Figure 3. The fact that this entropy exactly matches the difference between the total PMF and that arising from the brush distortion alone implies that $\Delta S^{\mathrm{comb}}(d)$ provides a full quantitative understanding of the effective interactions between a pair of NPs with palindromic sticky sites. It also clearly demonstrates that the strength of the sticky-sticky interaction (modeling the binding free energy between palindromic sticky strands) does not affect the strength of the interaction. Going further it also illustrates that the

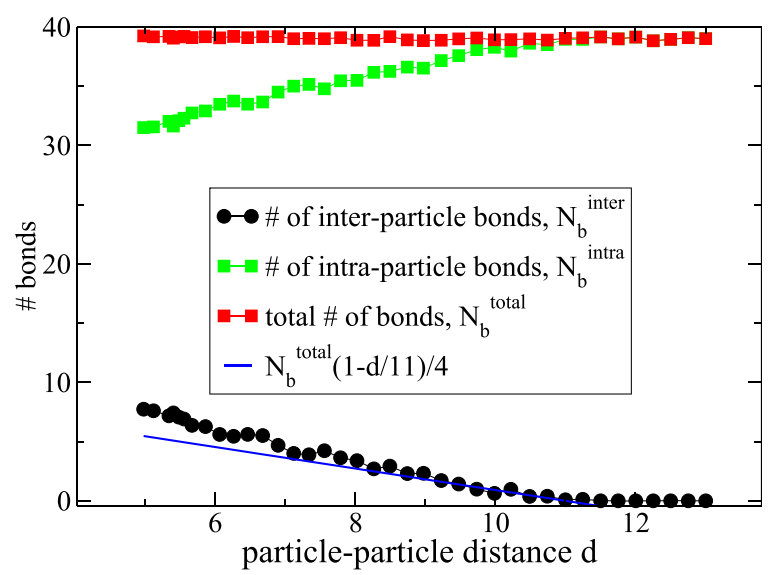

Figure 4. Interparticle distance dependence of the total number of bonds $N_{b}^{\text {total }}$ between sticky ends separated in its intra- $N_{b}^{\text {intra }}$ and inter- $N_{\mathrm{b}}^{\text {inter }}$ particle components. The blue line indicates the expected $N_{b}^{\text {inter }}$ assuming binding proportional to the overlapping surfaces of the two NPs of $\sim\left(1-d / d_{\text {max }}\right)$ (see text).

presence of attractive interactions between sticky ends across the two NPs does not cause any (measurable) change in the entropy of the brush layer. This result arises from the fact that the interactions between sticky beads for all pairs are identical, and hence there is no energetic reason to further distort the chains.

To probe this idea, we consider the distribution of chain ends and chain monomers as a function of distance $r$ from the center of the NP to which they belong. Figures 3 and 4 show that for distances $r$ greater than $\sim 11$, there are no inter-NP bonds. This implies that the maximum distance of the ends from the center of the NPs is 5.5. The distribution functions in Figure 5 clearly show that the chain ends are mainly confined

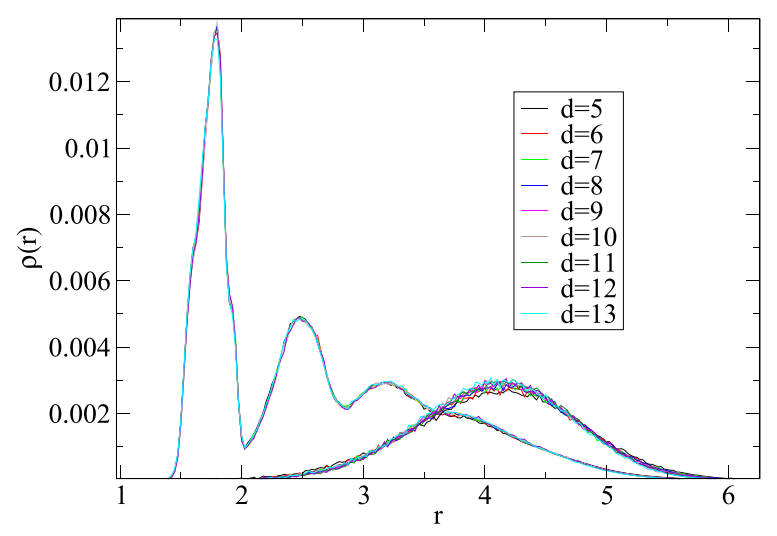

Figure 5. Distribution of monomers (left group of curves) and sticky ends (right group of curves) from the center of the NP to which these chains are bonded. The various curves correspond to a variety of inter-NP distances $d$, but all of the curves superpose within the uncertainties of the simulations.

to distances smaller than this value, providing an internal consistency check. The chain ends are predominantly localized to the "ends" of the brush, but there are ends present for all distances $\geq 2$. Before we use this distribution of chain ends to quantify the number of bonds formed between the two NPs (i.e., the data in Figure 4) we note that the distributions of chain ends, and also for the other chain monomers, are independent of the NP-NP separation. This argues that the 
chain conformations are unaltered when the NPs approach each other. We believe that the chains accommodate these constraints by essentially splaying along a direction normal to the vector joining the two centers without changing their conformations, e.g., by pivoting about the graft point.

For completeness we examine if and how the PMF can be modeled by known ideas in polymer physics. The repulsive part of the potential is expected to be similar to that proposed by Likos et al. for the case of star polymers. ${ }^{32,33}$ Indeed, in agreement with these ideas, we find that the far-field contribution (i.e., for $d \geq 8$ ) can be modeled by a Yukawa form as expected by these workers, suggesting that theories based on the repulsion between star molecules are sufficient to model this part of the inter-NP interaction. For shorter distances we find that a Gaussian dependence fits the data (see Methods section).

We now switch to understanding the variation of the number of bonds across different NPs, i.e., the data in Figure 4. The simplest model is to consider an Alexander brush, ${ }^{34}$ where the chain ends are at the edge of the brush. While this is a crude approximation relative to the simulation estimates in Figure 5, it is the easiest model to use in this context. We then accept the idea that chain conformations are not affected by the approach of the second NP and thus estimate that all the chain ends whose projections along the line joining NP centers are beyond the midpoint connecting the two NPs that are bonded. This simple model will yield that the number of bonds follows $\frac{N_{\mathrm{b}}^{\text {total }}}{4}\left(1-d / d_{\max }\right)$, where $d_{\max }$ indicates the distance at which interparticle bonds becomes possible. As shown in Figure 4, this model works almost quantitatively for $d \geq 8$, i.e., above the minimum in the PMF. For smaller values there are quantitative differences between the model predictions and simulation data. While we can use the known end distribution to have a better estimate of this quantity, we did not deem this as necessary since the fits, by definition, would be model sensitive and hence dependent on the details of the simulation parameters used.

Before concluding, we examine why these DNA-grafted colloids form crystal phases as against disordered liquids, both of which will be stabilized by these combinatorially driven attractions. Referring now to the Lennard-Jones potential, it is known that the triple point occurs when the reduced temperature is $\sim 0.6$, namely, when the attractive well depth of the potential is $\sim-1.67 k_{\mathrm{B}} T{ }^{35}$ All stronger attractions would lead to a direct coexistence between a gas phase and a solid. Our simulations (see also Methods section) suggest that, with only 42 grafted strands, the combinatorial attractive minimum is $\sim-2 k_{\mathrm{B}} T$. In the Methods, we show that this is strong enough to allow for the condensation of NPs into a dense phase and eventually to crystal formation. These results are, of course, strongly dependent on the number of grafts per NP and presumably the geometry of the NP, and thus we cannot unequivocally determine if liquids or crystals will form when these attractive interactions between the NPs becomes important.

We would also like to emphasize that the use of two NPs to determine effective interactions is likely to have significant corrections from three- and higher body interactions. In particular, we see that the grafted chains circumvent the potential conformational frustration that is introduced when two NPs approach each other by splaying the chains in a direction normal to the line joining the NP centers. This allows us to minimally perturb chain conformations, but this method of relieving frustration will not be available if additional NPs are present. How this effect modifies the effective NP interactions and thus our predictions for phase equilibria remains open.

The presence of this combinatorial attractive interaction between interacting NPs is reminiscent of the mean field ideas of Zilman et al., ${ }^{36}$ who examined the role of telechelic polymers on the phase behavior of microemulsion droplets. In that particular model, it was assumed that the end monomers preferred to locate themselves in the microemulsion droplets, while the remaining chain monomers preferred the (background) solvent. These workers then predicted an attractive interaction between the microemulsion droplets because some of the telechelic chains chose to bridge between two microemulsion droplets vs planting both ends in the same droplet. $^{37,38}$ Differently from Zilman et al. ${ }^{36}$ we compare accurately the PMF with the theoretical predictions, essentially without any fit parameter. The parallels of our work to Safran's idea for the emergence of attractive interactions between microemulsion droplets decorated by telechelic polymers or in the competition between branching and chaining ${ }^{39,40}$ emphasize the subtle but generalizable role of entropy in the assembly of heterogeneous materials with a "sticky" polymer constituent. We also note that when a particle is grafted with equal quantities of sticky ends $\mathcal{A}$ and its complementary sequence $\overline{\mathcal{A}}$, a phenomenology similar to the one we have discussed here is observed. ${ }^{23,24}$ Finally, we note that while we have based our study in the low- $T$ limit-to stress the independence of the attraction from the strength of the hybridization free energy of the palindromic sequence-the combinatorial entropy mechanism acts also when the number of bonds is controlled by temperature (or by salt concentration). This effect can be accounted for by including the binding probability (which depends on the salt concentration and temperature) as a prefactor in $N(d)$. We also note that the strength of the combinatorial entropy contribution can be modulated based on the DNA grafting density. One can also envision conditions in which the grafted strands are a mixture of palindromic and nonpalindromic sequences. In this case the combinatorial attraction would be complemented by the attraction generated by the finite concentration of nonpalidromic strands, providing a means to further tune the NP-NP interaction.

\section{CONCLUSIONS}

We use computer simulations to show that there are attractive interactions between pairs of NPs grafted with palindromic single-stranded DNA sequences. This contribution arises purely from combinatorial entropy, i.e., from the chains deciding if they want to pair with chains from the same vs different NPs. We evaluate theoretically and verify numerically this entropic contribution originating from the number of distinct bonding patterns associated with intra- and interparticle binding. This entropic attraction, which becomes more favorable with decreasing inter-NP distance, allows for the NP to form condensed phases in good agreement with experiments.

\section{METHODS/EXPERIMENTAL}

Experimental Details. The structures of the assembly were characterized by synchrotron-based small-angle X-ray scattering (SAXS) techniques. The SAXS experiments were conducted at the Complex Material Scattering beamline, National Synchrotron Light 
Source II. The scattering data were collected with a Pilatus area detector and converted to $1 \mathrm{D}$ scattering intensity $v s$ wave vector transfer, $q=(4 \pi / \lambda) \sin (\theta / 2)$, where $\lambda=0.918 \AA$ and $\theta$ are the wavelength of incident $\mathrm{X}$-ray and the scattering angle, respectively. The wave vector transfer was calibrated using silver behenate as a standard. The structure factor $S(q)$ was calculated as $I(q) / p(q)$, where $I(q)$ and $p(q)$ are background-corrected 1D scattering intensities extracted by angular averaging of images for the assembled system and dissociated particles, respectively.

Model Details. We model a nanoparticle grafted with DNA strands with a simple coarse-grained potential. The nanoparticle, the scaffold to which the DNA strands are grafted, is modeled as a rigid isosphere, ${ }^{8}$ created by splitting each triangle of a regular polyhedron into four smaller triangles, resulting in a tessellated sphere of 42 sites. The radius $R_{\mathrm{NP}}$ of the sphere is chosen as the unit of length. The 42 sites of the isosphere interact via a truncated and shifted LennardJones potential with $\sigma=0.63$ and $\epsilon=1.0$ up to distances $r=2^{1 / 6} \sigma$ (the position of the Lennard-Jones minimum). Each of the 42 interacting site is also bonded to its closest six neighbors via a FENE potential

$$
V_{\mathrm{FENE}}(r)=-\frac{K}{2} d_{\mathrm{FENE}}^{2} \ln \left[1-\left(\frac{r}{d_{\mathrm{FENE}}}\right)^{2}\right]
$$

with $K=100$ and $d_{\mathrm{FENE}}=0.742$.

A grafted DNA strand is modeled as a chain of seven identical monomers. The monomer-monomer and chain monomer-surface monomer interactions are modeled with a truncated and shifted Lennard-Jones potential with $\sigma=1.045$ and $\epsilon=1.0$. The polymer topology and the binding of the first monomer with the grafting site is enforced with the FENE potential, this time with $K=100$ and $d_{\mathrm{FENE}}=$ 1.056. Finally, the palindromic sticky end is represented as an additional monomer connected to the free end of each polymer. This sticky site interacts with other chain monomers and with surface monomers with a truncated and shifted Lennard-Jones potential this time with $\sigma=0.48$ and $\epsilon=1.0$. The sticky site is bonded to the seventh monomer with a FENE potential with $K=100$ and $d_{\mathrm{FENE}}=$ 0.54 . Hence, all interactions, except the sticky-sticky interaction, are repulsive. The interaction between sticky monomers is modeled by a short-range potential chosen such that all sticky ends pair at the temperature studied. The sticky end-sticky end interaction is modeled as

$$
V_{\text {sticky }}(r)=\epsilon_{\mathrm{s}}\left[\frac{1}{2}\left(\frac{\sigma_{\mathrm{ss}}}{r}\right)^{4}-1\right] \mathrm{e}^{\sigma_{\mathrm{ss}} /\left(r-r_{\mathrm{c}}\right)}
$$

with $\epsilon_{\mathrm{s}}=206.89, \sigma_{\mathrm{ss}}=0.3$, and $r_{\mathrm{c}}=1.5 \sigma_{\mathrm{ss}}$, resulting in a short-range potential with a minimum at $r_{\min }=0.3$ and depth $V_{\text {sticky }}^{\text {min }}=-14 k_{\mathrm{B}} T$. The potential vanishes at $r=r_{\mathrm{c}}$, and it is assumed to be zero for $r>r_{\mathrm{c}}$. The resulting interaction potentials are shown in Figure 6

To mimic the selectivity of the DNA sticky sequences and, at the same time, favor establishment of thermal equilibrium and proper sampling of several distinct bonding configurations, we implement the swap-algorithm described in ref 27 . This consists of adding a threebody repulsive interaction between close-by triplets of sticky sites. This three-body interaction is

$$
V_{\text {threebody }}=V_{\text {sticky }}^{\min } \sum_{i, j, k} V_{3}\left(r_{i j}\right) V_{3}\left(r_{i k}\right)
$$

where $V_{3}(r)=1$ for $r \leq r_{\text {min }}$ and $\frac{V_{\text {sticky }}(r)}{V_{\text {sticky }}^{\min }}$ for $r_{\min } \leq r \leq r_{\mathrm{c}}$. With this definition all terms $V_{3}(r)$ are between 0 and 1 , and correspondingly $V_{\text {threebody }}$ is positive and at most equal to $V_{\text {sticky }}^{\min }$ for each triplet. Thus, if a sticky site moves close to an existing bond, then the gain of forming one additional bond is compensated by the equally relevant repulsive three-body term. The swap process thus takes place on an essentially flat potential energy surface. The three-body potential also significantly discourages the formation of aggregates formed by three or more sticky sites due to the overwhelming resulting

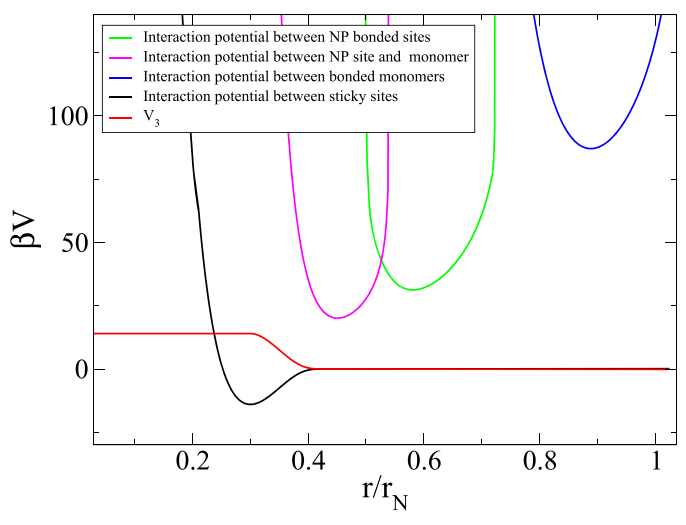

Figure 6. Graphic representation of the different interaction potentials used in the present study. The combination of the truncated and shifted Lennard-Jones potential with the FENE potential results in an anharmonic minimum that defines the bonding distance. The potential $V_{3}$ is defined in the text. It has been multiplied by $V_{\text {sticky }}^{\min }$ for clarity.

repulsion, effectively implementing the single-bond per patch condition, an essential condition to model the specificity of the DNA association. We finally note that the evaluation of $V_{\text {threebody }}$ does not require significant additional calculations, since it is expressed in terms of already calculated pair interaction energies.

Second Virial Coefficient of the Effective Potential. From the effective potential, it is possible to evaluate the second virial c o e ffi cie n t $\quad B_{2}(T), \quad$ d e fin ed a s $\left.B_{2}=-2 \pi \int_{0}^{\infty} r^{2}\left(\mathrm{e}^{-\beta V_{\text {eff }}(r)}-1\right)\right) d r \approx 5 \times 10^{3} . B_{2}$ does not depend on temperature since it is based on an entropic effective potential. At the level of $B_{2}$, the equation of state, $\beta P=\rho+B_{2} \rho^{2}$, has a maximum at $\rho=-1 / 2 B_{2} \approx 10^{-4}$.

Gas-Liquid Phase Coexistence. Starting from the numerically evaluated effective potential (Figure 2), we have evaluated the gasliquid coexistence by performing Monte Carlo calculations in the grand canonical ensemble, fixing $k_{\mathrm{B}} T=1, L=60$ (where $L$ is the side of the cubic box simulated), and fixed chemical potential $\mu$. The probability of finding $N$ particles in the system is then given by

$$
P(N)=\frac{z^{N} Q(V, T, N)}{\sum_{N=0}^{\infty} z^{N} Q(V, T, N)}
$$

where $z=\mathrm{e}^{\beta \mu}, V=L^{3}$, and $Q(V, T, N)$ is the canonical partition function of a system with $N$ particles. The distribution $P(N)$ is unimodal in thermodynamically stable states (and in this case the equilibrium density is $\left.\rho=\sum N P(N) / V\right)$. When $\mu$ describes a coexistence state, then $P(N)$ has two equal area peaks, one describing the gas and one describing the liquid state. The two coexisting densities can be estimated by averaging $N$ limited to the area under each peak.

To evaluate $P(N)$ efficiently, we have implemented the successive umbrella sampling technique. In this methodology, the explored range of $N$ values $\left(0<N<N_{\max }\right)$ is partitioned into $N_{\text {nmax }}$ subranges $\left[N_{i}, N_{i}\right.$ $+1]$, with $0<N_{i}<N_{\max }$. Then $N_{\mathrm{nmax}}$-independent GCMC simulations at the same $z$ are performed, each of them constrained to explore only the interval $\left[N_{i}, N_{i}+1\right]$ to generate accurate values of the ratio $P\left(N_{i}+\right.$ 1) $/ P\left(N_{i}\right)$. Splicing together all ratios and properly normalizing the resulting distribution $\left(\sum P(N)=1\right)$ provides the requested distribution. This method is particularly suited for large CPU clusters, where each node explores its own $\left[N_{i}, N_{i}+1\right]$ interval. We also note that histogram reweighing techniques may be applied to optimize the value of $z$ and detect coexistence, especially because we have the entire $N$ dependence. We also note that this method is particularly suited to study phase coexistence since large barriers, if present, are also split among several $\left[N_{i j} N_{i}+1\right]$ intervals. 
The resulting $P(N)$ for $\beta \mu=-7.478$ is shown in Figure 7. The density of the gas phase is $5 \times 10^{-6}$, while the density of the liquid phase is $2 \times 10^{-3}$.

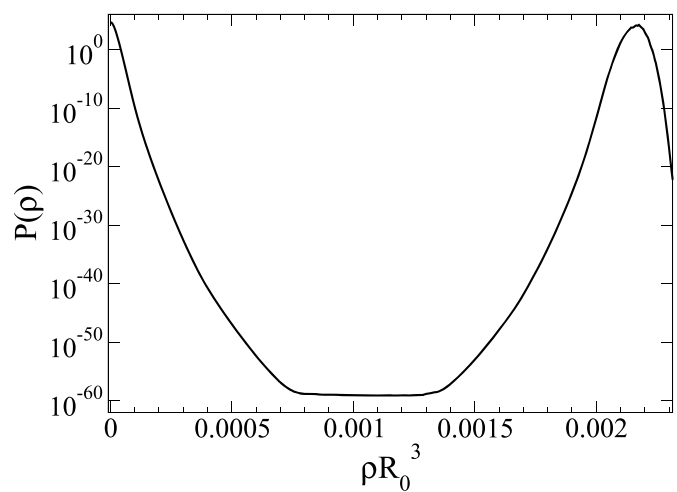

Figure 7. Distribution of the density fluctuations observed in a grand canonical simulation at the gas-liquid coexistence chemical potential. The cubic simulation box has side $60 R_{\mathrm{NP}}$, where $R_{\mathrm{NP}}$ is the particle radius, corresponding to about 500 particles at the highest reported density. Note the 60 or more order of magnitude variation of $P(N)$ is made possible by the use of the successive umbrella sampling method.

Effective Potential in the Absence of Sticky Interactions. To extract the role of the combinatorial entropy in the effective potential, we have also simulated the same model described above but setting the amplitude of the sticky-sticky interaction at zero $\left(\epsilon_{s}=0\right)$. The resulting purely repulsive potential is shown in Figure 2. Here we show possible parametrization of the resulting potential based on two functional forms. A reasonable global fit, but which does poorly in the regime of interest, i.e., for $\beta V_{\text {eff }}<5$, is offered by the Gaussian function

$$
\beta V_{\text {eff }}=A_{\mathrm{G}} \mathrm{e}^{-r^{2} / 2 \sigma_{\mathrm{g}}^{2}} \text { with } A_{\mathrm{G}}=320 \sigma_{\mathrm{g}}=6.54
$$

The region $\beta V_{\text {eff }}<5$ is instead well-represented by a Yukawa functional form, the characteristic functional form describing star polymers: $^{33}$

$$
\beta V_{\text {eff }}=A_{Y} \frac{\mathrm{e}^{-r / \sigma_{Y}}}{r} \text { with } A_{Y}=5.83 \times 10^{6} \sigma_{Y}=0.63
$$

The quality of the fit is shown in Figure 8 .

Numerical Details. Umbrella Sampling. To evaluate the effective potential, we have performed simulations of the model described here with an additional harmonic contribution constraining

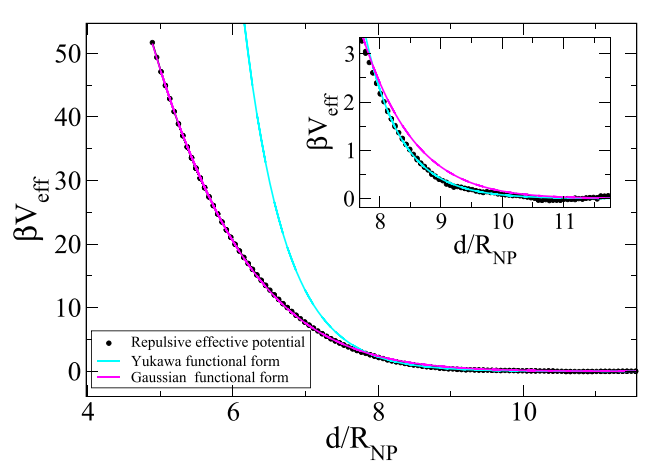

Figure 8. Analysis of the distance dependence of the repulsive potential originating from the polymer-polymer interactions. The region $\beta V_{\text {eff }}(d)<3$ is well represented by a Yukawa decay. The region between $3<\beta V_{\text {eff }}(d)<50$ can be well described by a Gaussian functional form. the particle-particle distance around a fixed value. Specifically, we have added the potential

$$
V_{\mathrm{US}}=\frac{1}{2} K_{\mathrm{US}}\left(d-d_{\mathrm{US}}\right)^{2}
$$

and explored 38 distinct values of $d_{\mathrm{Us}}$. Figure 9 shows the evolution of the distance between the two particles during the simulation, for all 38

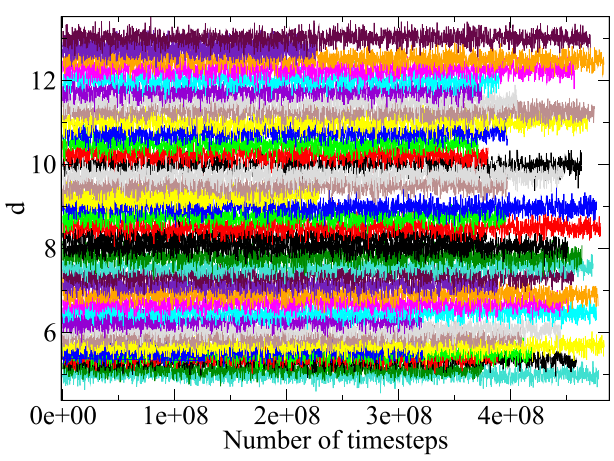

Figure 9. Evolution in time of the distance between the two NPs, for all 38 runs, differing in the value of $d_{\mathrm{Us}}$. The significant overlap between adjacent values of $d_{\mathrm{US}}$ allows us to extract the effective potential.

independent runs. For all runs $\beta K_{\mathrm{US}}=100$. The selected values of $d_{\mathrm{US}}$ are $4.3,4.5,4.8,4.9,5.0,5.1,5.25,5.5,5.75,6.0,6.25,6.5,6.75,7.0$, $7.25,7.5,7.75,8.0,8.25,8.5,8.75,9.0,9.25,9.50,9.75,10.0,10.25$, $10.5,10.75,11.0,11.25,11.5,11.75,12.0,12.25,12.50,12.75$, and 13.00. By combining together the 38 histograms $P(d)$, the effective potential, can be calculated implementing standard methodologies. ${ }^{31}$

\section{AUTHOR INFORMATION}

\section{Corresponding Author}

Francesco Sciortino - Department of Physics, Sapienza Universita' di Roma, 00185 Rome, Italy; 이이.org/00000002-2418-2713; Email: francesco.sciortino@uniroma1.it

\section{Authors}

Yugang Zhang - Center for Functional Nanomaterials, Brookhaven National Laboratories, Upton, New York 11973, United States

Oleg Gang - Department of Chemical Engineering and Department of Applied Physics and Applied Mathematics, Columbia University, New York, New York 10027, United States; Center for Functional Nanomaterials, Brookhaven National Laboratories, Upton, New York 11973, United States

Sanat K. Kumar - Department of Chemical Engineering, Columbia University, New York, New York 10027, United States; ○ orcid.org/0000-0002-6690-2221

Complete contact information is available at: https://pubs.acs.org/10.1021/acsnano.9b10123

\section{Notes}

The authors declare no competing financial interest.

\section{ACKNOWLEDGMENTS}

S.K. thanks the Department of Energy (DE-SC0008772) for funding this research activity. F.S. acknowledges support from MIUR-PRIN (Grant No. 2017Z55KCW) and from Regione Lazio (Grant No. 85857-0051-0085). Some of this work was performed when F.S. was visiting Columbia University. F.S. thanks CU for their hospitality. We acknowledge discussions with Alexei V. Tkachenko. This research used resources of the 
Center for Functional Nanomaterials, supported by U.S. DOE Office of Science Facilities at Brookhaven National Laboratory under Contract No. DE-SC0012704.

\section{REFERENCES}

(1) Nykypanchuk, D.; Maye, M. M.; Van Der Lelie, D.; Gang, O. DNA-guided crystallization of colloidal nanoparticles. Nature 2008, $451,549$.

(2) Park, S. Y.; Lytton-Jean, A. K.; Lee, B.; Weigand, S.; Schatz, G. C.; Mirkin, C. A. DNA-programmable nanoparticle crystallization. Nature 2008, 451, 553.

(3) Mirkin, C. A.; Letsinger, R. L.; Mucic, R. C.; Storhoff, J. J. A DNA-based method for rationally assembling nanoparticles into macroscopic materials. Nature 1996, 382, 607.

(4) Alivisatos, A. P.; Johnsson, K. P.; Peng, X.; Wilson, T. E.; Loweth, C. J.; Bruchez, M. P., Jr; Schultz, P. G. Organization of nanocrystal molecules' using DNA. Nature 1996, 382, 609.

(5) Wang, Y.; Wang, Y.; Zheng, X.; Ducrot, É.; Yodh, J. S.; Weck, M.; Pine, D. J. Crystallization of DNA-coated colloids. Nat. Commun. 2015, 6, 7253.

(6) Dreyfus, R.; Leunissen, M. E.; Sha, R.; Tkachenko, A. V.; Seeman, N. C.; Pine, D. J.; Chaikin, P. M. Simple quantitative model for the reversible association of DNA coated colloids. Phys. Rev. Lett. 2009, 102, No. 048301.

(7) Varilly, P.; Angioletti-Uberti, S.; Mognetti, B. M.; Frenkel, D. A general theory of DNA-mediated and other valence-limited colloidal interactions. J. Chem. Phys. 2012, 137, No. 094108.

(8) de Pablo, J. J. Coarse-grained simulations of macromolecules: from DNA to nanocomposites. Annu. Rev. Phys. Chem. 2011, 62, $555-574$

(9) Lequieu, J. P.; Hinckley, D. M.; de Pablo, J. J. A molecular view of DNA-conjugated nanoparticle association energies. Soft Matter 2015, 11, 1919-1929.

(10) Halverson, J. D.; Tkachenko, A. V. DNA-programmed mesoscopic architecture. Phys. Rev. E 2013, 87, No. 062310.

(11) Angioletti-Uberti, S.; Mognetti, B. M.; Frenkel, D. Theory and simulation of DNA-coated colloids: a guide for rational design. Phys. Chem. Chem. Phys. 2016, 18, 6373-6393.

(12) Mognetti, B. M.; Varilly, P.; Angioletti-Uberti, S.; MartinezVeracoechea, F. J.; Dobnikar, J.; Leunissen, M. E.; Frenkel, D. Predicting DNA-mediated colloidal pair interactions. Proc. Natl. Acad. Sci. U. S. A. 2012, 109, E378-E379.

(13) Tkachenko, A. V. Morphological diversity of DNA-colloidal self-assembly. Phys. Rev. Lett. 2002, 89, 148303.

(14) Macfarlane, R. J.; Lee, B.; Jones, M. R.; Harris, N.; Schatz, G. C.; Mirkin, C. A. Nanoparticle superlattice engineering with DNA. Science 2011, 334, 204-208.

(15) Biancaniello, P. L.; Kim, A. J.; Crocker, J. C. Colloidal interactions and self-assembly using DNA hybridization. Phys. Rev. Lett. 2005, 94, No. 058302.

(16) Kim, A. J.; Biancaniello, P. L.; Crocker, J. C. Engineering DNAmediated colloidal crystallization. Langmuir 2006, 22, 1991-2001.

(17) Tian, Y.; Lhermitte, J. R.; Bai, L.; Vo, T.; Xin, H. L.; Li, H.; Li, R.; Fukuto, M.; Yager, K. G.; Kahn, J. S.; Xiong, Y.; Binevich, B.; Kumar, S. K.; Gang, O. Ordered three-dimensional nanomaterials using DNA-prescribed and valence-controlled material voxels. Nat. Mater. 2020, 1-8.

(18) Tan, S. J.; Kahn, J. S.; Derrien, T. L.; Campolongo, M. J.; Zhao, M.; Smilgies, D.-M.; Luo, D. Crystallization of DNA-Capped Gold Nanoparticles in High-Concentration, Divalent Salt Environments. Angew. Chem., Int. Ed. 2014, 53, 1316-1319.

(19) Lequieu, J.; Córdoba, A.; Hinckley, D.; de Pablo, J. J. Mechanical response of dna-nanoparticle crystals to controlled deformation. ACS Cent. Sci. 2016, 2, 614-620.

(20) Escobedo, F. A. Engineering entropy in soft matter: The bad, the ugly and the good. Soft Matter 2014, 10, 8388-8400.

(21) Sciortino, F. Entropy in self-assembly. Riv. Nuovo Cimento 2019, 42, 511-548.
(22) Zhang, Y.; Pal, S.; Srinivasan, B.; Vo, T.; Kumar, S.; Gang, O. Selective transformations between nanoparticle superlattices via the reprogramming of DNA-mediated interactions. Nat. Mater. 2015, 14, 840-847.

(23) Angioletti-Uberti, S.; Mognetti, B. M.; Frenkel, D. Re-entrant melting as a design principle for DNA-coated colloids. Nat. Mater. 2012, 11, 518 .

(24) Bachmann, S. J.; Kotar, J.; Parolini, L.; Šarić, A.; Cicuta, P.; Di Michele, L.; Mognetti, B. M. Melting transition in lipid vesicles functionalised by mobile DNA linkers. Soft Matter 2016, 12, 78047817.

(25) Lukatsky, D.; Frenkel, D. Surface and bulk dissolution properties, and selectivity of DNA-linked nanoparticle assemblies. J. Chem. Phys. 2005, 122, 214904.

(26) Leunissen, M. E.; Frenkel, D. Numerical study of DNAfunctionalized microparticles and nanoparticles: Explicit pair potentials and their implications for phase behavior. J. Chem. Phys. 2011, 134, No. 084702.

(27) Sciortino, F. Three-body potential for simulating bond swaps in molecular dynamics. Eur. Phys. J. E: Soft Matter Biol. Phys. 2017, 40, 3.

(28) Rovigatti, L.; Nava, G.; Bellini, T.; Sciortino, F. Self-dynamics and collective swap-driven dynamics in a particle model for vitrimers. Macromolecules 2018, 51, 1232-1241.

(29) Oyarzún, B.; Mognetti, B. M. Efficient sampling of reversible cross-linking polymers: Self-assembly of single-chain polymeric nanoparticles. J. Chem. Phys. 2018, 148, 114110.

(30) Oyarzún, B.; Mognetti, B. M. Programming configurational changes in systems of functionalised polymers using reversible intramolecular linkages. Mol. Phys. 2018, 116, 2927-2941.

(31) Frenkel, D.; Smit, B. Understanding Molecular Simulation: From Algorithms to Applications; Elsevier, 2001; Vol. 1.

(32) Daoud, M.; Cotton, J. Star shaped polymers: a model for the conformation and its concentration dependence. J. Phys. 1982, 43, $531-538$.

(33) Likos, C.; Löwen, H.; Watzlawek, M.; Abbas, B.; Jucknischke, O.; Allgaier, J.; Richter, D. Star polymers viewed as ultrasoft colloidal particles. Phys. Rev. Lett. 1998, 80, 4450.

(34) Rabin, Y.; Alexander, S. Stretching of grafted polymer layers. Europhys. Lett. 1990, 13, 49.

(35) Hansen, J.-P.; McDonald, I. R. Theory of Simple Liquids; Elsevier, 1990.

(36) Zilman, A.; Kieffer, J.; Molino, F.; Porte, G.; Safran, S. Entropic phase separation in polymer-microemulsion networks. Phys. Rev. Lett. 2003, 91, No. 015901.

(37) Molino, F.; Appell, J.; Filali, M.; Michel, E.; Porte, G.; Mora, S.; Sunyer, E. A transient network of telechelic polymers and microspheres: structure and rheology. J. Phys.: Condens. Matter 2000, 12, A491.

(38) Filali, M.; Michel, E.; Mora, S.; Molino, F.; Porte, G. Stress relaxation in model transient networks: percolation and rearrangement of the crosslinks. Colloids Surf., A 2001, 183, 203-212.

(39) Zilman, A.; Tlusty, T.; Safran, S. Entropic networks in colloidal, polymeric and amphiphilic systems. J. Phys.: Condens. Matter 2003, 15 , S57.

(40) Russo, J.; Tavares, J. M.; Teixeira, P. I. C.; da Gama, M. T.; Sciortino, F. Reentrant phase diagram of network fluids. Phys. Rev. Lett. 2011, 106, No. 085703. 